\title{
Correction to: Analysis of QTL-allele system conferring drought tolerance at seedling stage in a nested association mapping population of soybean [Glycine max (L.) Merr.] using a novel GWAS procedure
}

Mueen Alam Khan ${ }^{1,2} \cdot$ Fei Tong ${ }^{1,2} \cdot$ Wubin Wang ${ }^{1,2,3,4,5} \cdot$ Jianbo He $e^{1,2,3,4} \cdot$ Tuanjie Zhao ${ }^{1,2,3,4,5} \cdot$ Junyi Gai $^{1,2,3,4,5}$

Published online: 20 March 2019

(c) Springer-Verlag GmbH Germany, part of Springer Nature 2019

Correction to: Planta (2018) 248:947-962

https://doi.org/10.1007/s00425-018-2952-4

In the third sentence of "SNP calling and SNPLDB assembly" in "Materials and methods" of the published manuscript, four numbers related to the DNA sequencing data are not correct. The error does not affect any results and conclusions of the article. The four incorrect numbers are 1144.56, 110.87, 3.86 and 4.57, while the correct numbers should be 1219.6, 101.1, $4.4 \times$ and 3.7, respectively, and the correct sentence is given below.

The sequences of the 403 lines were obtained using Illumina HiSeq 2000 instrument through the multiplexed shotgun genotyping method (Andolfatto et al. 2011) with the DNA fragments between 400 and $600 \mathrm{bp}$, generating a total of the 1219.6 million paired end reads of $90 \mathrm{bp}$ (including 6-bp index) in length (101.1 Gb of the sequence) approximately $4.4 \times$ in depth and $3.7 \%$ coverage.

The original article can be found online at https://doi.org/10.1007/ s00425-018-2952-4.

Junyi Gai

sri@njau.edu.cn

1 Soybean Research Institute, Nanjing Agricultural University, Nanjing 210095, Jiangsu, China

2 National Center for Soybean Improvement, Ministry of Agriculture, Nanjing 210095, Jiangsu, China

3 Key Laboratory of Biology and Genetic Improvement of Soybean, Ministry of Agriculture, Nanjing 210095, Jiangsu, China

4 National Key Laboratory for Crop Genetics and Germplasm Enhancement, Nanjing 210095, Jiangsu, China

5 Jiangsu Collaborative Innovation Center for Modern Crop Production, Nanjing Agricultural University, Nanjing 210095, Jiangsu, China 